\title{
Cicatrização de ferida profunda em equino com uso de dreno ativo, laser de baixa potência e ultrassom terapêutico
}

\section{Deep wound healing in horses using open drain, low level laser therapy, and therapeutic ultrasound}

\author{
Gustavo Henrique Marques Araujo ${ }^{1^{*}}$, Anna Beatriz Borges de Carvalho ${ }^{1}$, Guilherme Pinheiro Santos ${ }^{1}$, Jordana \\ Cabral Rosa dos Anjos², Rhavilla Santos de Oliveira ${ }^{1}$, Rogério Elias Rabelo ${ }^{1}$, Júlia Miranda de Moraes ${ }^{1}$ \\ ${ }^{1}$ Universidade Federal de Goiás (UFG), Jataí, GO, Brasil \\ ${ }^{2}$ Universidade Federal de Uberlândia (UFU), Uberlândia, MG, Brasil
}

\section{Resumo}

As lesões cutâneas em equinos possuem grande destaque na área científica por apresentarem cicatrização lenta, com formação de tecido de granulação exuberante. Várias linhas de pesquisa buscam a aplicação de novas técnicas para melhorar o processo de reparação tecidual e a qualidade final do tecido cicatricial, como o uso do laser de baixa potência (LBP) e o ultrassom terapêutico (UST). Estes estimulam a produção de fibroblastos e de colágeno, o que resulta em aumento da força de tensão da ferida e redução do tempo de cicatrização. Assim, devido à importância clínica das lesões cutâneas nessa espécie, o presente trabalho teve como objetivo relatar um caso de laceração cutânea em equino apresentando lesão cutânea exsudativa, tratado com drenagem ativa, LBP e UST. Optou-se pela cicatrização por segunda intenção, com uso de dreno ativo e curativos diários. Fezse a aplicação de LBP (20 Joules/ $\mathrm{cm}^{2}$ em duas aplicações e 10 Joules $/ \mathrm{cm}^{2}$ nas demais sessões) nas bordas da ferida de forma pontual, em pontos equidistantes de $1 \mathrm{~cm}$, e em varredura sobre o leito da ferida. O UST foi aplicado pelo método direto, na presença de gel sobre a pele tricotomizada, nas linhas de tensão da ferida e nas áreas de formação de fibrose e aderência, com frequência de $3 \mathrm{MHz}$, intensidade de pulso de $100 \mathrm{~Hz}$ a $10 \%$, intensidade de $0,5 \mathrm{~W} / \mathrm{cm}^{2}$ no modo pulsado, por 12 minutos. Após 90 dias do início do tratamento, a ferida apresentava $7,6 \mathrm{~cm}^{2}$, demonstrando redução de $81 \%$ da ferida inicial, com nivelamento plano, boa retração de bordas, formação adequada de tecido epitelial e redução do tecido de granulação. A combinação das duas técnicas foi satisfatória, diminuindo consideravelmente o tempo de cicatrização e apresentando interessante resultado estético e cicatricial.

Palavras-chave: Cavalo. Colágeno. Fotobiomodulação. Fisioterapia. Reabilitação. 


\section{Abstract}

Equine cutaneous lesions are one of the highlights in scientific research due to their slow healing process and exuberant granulation tissue formation. Plenty of research branches aim to apply new techniques in order to improve both tissue repair process and scar tissue quality, like low level laser therapy (LLLT) and therapeutic ultrasound (TUS). Those stimulate the production of fibroblasts and collagen, increasing wound tensile strength, and speeding up the healing process. Thereby, due to the clinical relevance of the equine cutaneous lesions studies, this work meant to report a case of laceration in a horse, presenting exudative skin lesion, treated with active drainage, LLLT and TUS. Second intention wound healing with active drain and daily dressings was the chosen treatment option. LLLT was applied (20 Joules $/ \mathrm{cm}^{2}$ in the first two sessions and 10 Joules $/ \mathrm{cm}^{2}$ in the ones that followed) on the border of the wound in equidistant points of $1 \mathrm{~cm}$, and sweept over the wound bed. Through direct contact method, TUS was applied directly over tricothomized skin with gel, on the tension lines of the wound and on the areas of fibrosis formation and adherence, with frequency of $3 \mathrm{MHz}$, pulse intensity of $100 \mathrm{~Hz}$ at $10 \%$, intensity of $0,5 \mathrm{~W} / \mathrm{cm}^{2}$ in pulse mode for 12 minutes. Ninety days after the beginning of the treatment, the wound was 81\% smaller, measuring $7,6 \mathrm{~cm}^{2}$, leveled, good border retraction, proper epithelial tissue formation, and granulation tissue reduction. The combination of the two techniques provided satisfactory results, considerably reducing healing time, with interesting aesthetic and healing achievements.

Keywords: Horse. Collagen. Photobiomodulation. Physiotherapy. Rehabilitation.

\section{Introdução}

A lesão cutânea é uma das afecções mais comumente encontradas na clínica de equinos. Fatores como o comportamento intempestivo frente à manipulação, ou mesmo devido à presença de materiais pontiagudos ou cortantes e instalações inadequadas nos locais de manejo, predispõe à ocorrência de lesões de pele (Viana et al., 2014). Os cavalos possuem características particulares frente aos processos orgânicos de cicatrização de feridas, despertando um importante interesse clínico, científico e econômico sobre o assunto (Araujo et al., 2017). Nas feridas tratadas por segunda intenção, são frequentemente observadas alterações como a contaminação da lesão, com produção de conteúdo purulento, cicatrização mais lenta em comparação às outras espécies, além da formação excessiva de tecido de granulação. Todas essas alterações são apontadas como desafios para o profissional médico veterinário, necessitando, assim, da aplicação de metodologias específicas para a melhora do processo cicatricial (Paganela et al., 2009; Moraes et al., 2014a).

Dessa forma, nas feridas contaminadas e de caráter exsudativo, a técnica de drenagem passiva ou ativa pode ser empregada. Os drenos ativos constituem a aplicação de um tubo fenestrado sobre o tecido a ser tratado, acoplado a um sistema a vácuo para sucção do conteúdo. Esse tipo de técnica tem como vantagem apresentar uma drenagem contínua do fluido, enquanto houver vácuo, além de impedir o movimento retrógado de contaminantes para o interior da ferida (Celeste, 2017).

Adicionalmente, com o objetivo de acelerar o processo cicatricial e melhorar a qualidade final de granulação e tensão de uma ferida, o laser de baixa potência (LBP) e o ultrassom terapêutico (UST) constituem metodologias de tratamento não invasivas, preconizadas em diversas situações na clínica de equinos. Ambos estimulam o desenvolvimento de fibroblastos e a produção de colágeno (Moraes et al., 2010; Moraes et al., 2014b; Diwan e Abid, 2016; Alkahtani et al., 2017).

0 LBP é um tipo de radiação eletromagnética com ação principal nas mitocôndrias e membrana celular. Gera aumento de ATP, modificação do transporte iônico e transcrição e replicação do DNA (Moraes et al., 2014b; Diwan e Abid, 2016). Já o UST é uma onda sonora propagativa com efeito mecânico sobre as células, resultando em excitação celular, aumento do metabolismo e da atividade celular. As consequências diretas para feridas cutâneas incluem o estímulo da atividade de fibroblastos, aumento da angiogênese, aumento na síntese de proteínas, redução da dor e melhora da força de tensão da ferida. Assim, o LBP e o UST são capazes de promover o aumento da atividade morfofuncional, da microcirculação e oxigenação 
tecidual (Moraes et al., 2014a, b; Diwan e Abid, 2016; Alkahtani et al., 2017; Chang et al., 2017).

Nesse contexto, o presente trabalho tem por objetivo relatar a reconstituição tecidual de lesão cutânea lacerativa em um equino, com a utilização de dreno ativo em associação com LBP e UST.

\section{Relato do caso}

Um equino, macho, de aproximadamente 4 anos de idade, clinicamente debilitado e apresentando lesão cutânea lacerativa extensa, foi atendido no Setor de Cirurgia de Grandes Animais da Universidade Federal de Goiás, em Jataí, GO. A lesão se localizava no flanco esquerdo, cranialmente à região inguinal e lateralmente ao prepúcio. Apresentava profundidade acentuada, exsudato purulento, hemorragia, bordos irregulares, elevados, edematosos e invaginados (Figura 1). 0 proprietário não soube informar o evento desencadeante da lesão, e a procura por atendimento ocorreu cinco dias após a identificação.

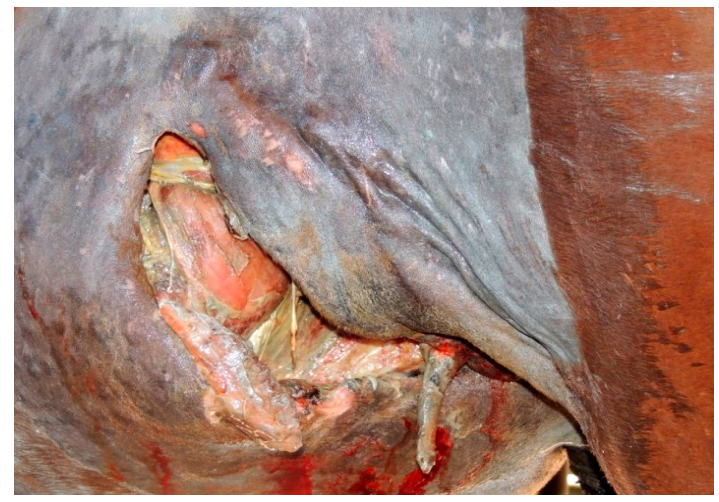

Figura 1 - Aspecto e área da ferida em equino antes do tratamento cirúrgico.

Optou-se pelo acompanhamento e cicatrização por segunda intenção, higienizando a ferida com líquido de Dakin e clorexidine, antibioticoterapia (penicilina procaína $30.000 \mathrm{UI} / \mathrm{Kg}$, q 24h, IM) e anti-inflamatório não esteroidal (AINE) (fenilbutazona $4,4 \mathrm{mg} / \mathrm{Kg}$, q $24 \mathrm{~h}$, IV). Os curativos foram feitos duas vezes ao dia e pretendiam combater a infecção da ferida, além de proporcionar um ambiente favorável para cicatrização, com remoção de sujidades, tecidos necróticos e manutenção da umidade ideal.

Além da terapia medicamentosa, optou-se pelo uso do dreno ativo durante seis dias, facilitando a manutenção da limpeza da ferida e excreção do conteúdo acumulado. A terapia com LBP e UST ocorreu em duas etapas.

A primeira etapa foi iniciada no sétimo dia após o atendimento inicial, no qual se aplicou o LBP (Physiolux dual, $830 \mathrm{~nm}$ de comprimento de onda, Arsenieto de Gálio e Alumínio-AsGaAl, potência de $40 \mathrm{~mW}$ - Bioset, Rio Claro, Brasil) com 20 Joules/ $\mathrm{cm}^{2}$ de intensidade (energia de saída do aparelho), nas bordas da ferida, de forma pontual, com intervalos equidistantes de $1 \mathrm{~cm}$, e em varredura total sobre a borda da ferida, totalizando duas aplicações de LBP. Com o preenchimento da ferida pelo tecido de granulação e início da epitelização, após 37 dias do primeiro atendimento, iniciou-se a segunda fase de tratamento. Nessa etapa, a terapia com LBP foi retomada, aplicando-se 10 Joules/ $\mathrm{cm}^{2}$ nas bordas, associada ao UST (Sonacel Dual Bioset, São Paulo, Brasil). O UST foi procedido pelo método direto, na presença de gel de contato sobre a pele tricotomizada, com frequência de $3 \mathrm{MHz}$ no modo pulsado (não-térmico), intensidade de 0,5 $\mathrm{W} / \mathrm{cm}^{2}$ e intensidade de pulso de $100 \mathrm{~Hz}$ a $10 \%$. Permaneceu-se com o transdutor sobre as linhas de tensão da ferida, nas áreas de formação de fibrose e aderências por 12 minutos, somando-se cinco aplicações a cada dois dias.

Conforme ocorria a retração centrípeta da ferida, diminuía-se o tempo de aplicação do UST. Mensurava-se a ferida com uma escala padrão e esta era fotografada com câmera digital de alta resolução, com intuito de acompanhar a regressão centrípeta dos bordos. As imagens foram analisadas no programa de imagens Image J versão 1.3.1 (NIH, EUA), obtendo-se as áreas da lesão em centímetros quadrados.

\section{Resultados}

No ato do atendimento, a ferida apresentavase de grande extensão, percorrendo desde a área lateral ao prepúcio até a região inguinal, com área 
superior a $60 \mathrm{~cm}^{2}$ e de profundidade acentuada (Figura 1), o que dificultou a mensuração exata da área da ferida. Após 37 dias de tratamento com o LBP, ao início da segunda etapa, a ferida possuía a extensão de $39,9 \mathrm{~cm}^{2}$, com uma redução de aproximadamente $50 \%$ do seu tamanho inicial, apresentando-se plana, com granulação superficial e com início de epitelização de bordos (Figura 2). Após 90 dias do início do tratamento, a ferida se apresentava com $7,6 \mathrm{~cm}^{2}$, demonstrando redução de $81 \%$ em sua extensão total inicial. As aplicações de LBP e UST foram finalizadas mesmo sem a total retração da ferida, pois esta se encontrava em estágio final de cicatrização, com boa epitelização e retração de bordas e ausência de tecido de granulação exuberante (Figura 3).

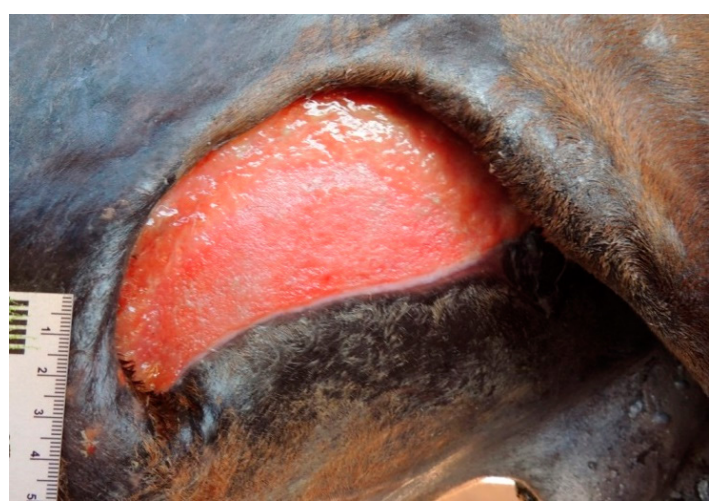

Figura 2 - Aspecto e área da ferida em equino 37 dias após 0 início do tratamento.

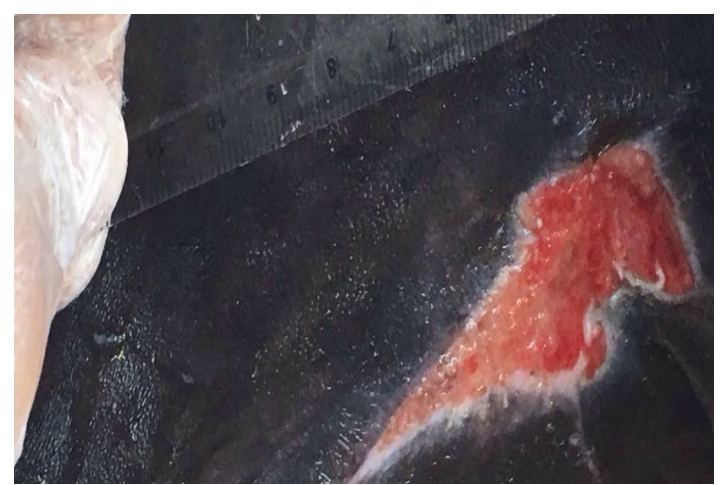

Figura 3 - Aspecto e área da ferida em equino ao final do tratamento, combinando dreno ativo, laser de baixa potência e ultrassom terapêutico.

\section{Discussão}

Segundo Rashidi et al. (2015), Diwan e Abid (2016) e Chang et al. (2017), as interações do LBP e do UST com os tecidos geram efeitos como alteração de potenciais de membrana celular com aumento do metabolismo, estimulação da angiogênese, da motilidade e da taxa de proliferação celular, incluindo fibroblastos e células do sistema imunológico. Isso gera acréscimo da produção de colágeno local com consequente aumento do tecido de granulação, conforme observado na ferida relatada, em que houve superficialização da ferida e possibilidade de epitelização após a utilização de LBP e, posteriormente, de UST. Além da melhora do processo cicatrial, Chang et al. (2017) relataram a atuação do UST na interrupção do biofilme bacteriano em lesões, por meio de um debridamento físico pelas ondas vibratórias, além da bioestimulação da resposta imunológica local e fibrinólise, o que pode ser favorável na utilização da terapia em tecidos com alta taxa de contaminação e exsudação.

A biomodulação dos processos inflamatórios e o maior desempenho de células fagocitárias também são características da ação do LBP (Rashidi et al., 2015; Diwan e Abid, 2016). Moraes et al. (2014b) utilizaram LBP e UST em feridas sépticas de equinos, e observaram tanto o controle da exsudação purulenta como a intensa produção de tecido de granulação e retração das bordas da ferida, corroborando com os autores acima citados e com o caso em questão, com atuação de ambas as terapias para a melhora da qualidade do processo cicatricial e controle da infecção local.

Ainda, Rashidi et al. (2015) relataram que ensaios clínicos têm sido conduzidos com o intuito de aplicação do LBP em feridas crônicas em pacientes diabéticos, devido ao alto grau de necrose e desenvolvimento de feridas cutâneas, o que comprova ainda mais a eficácia do método em estímulação do metabolismo celular e angiogênese, eliminando a hipóxia e a isquemia tecidual (Chang et al., 2017). Apesar de todos os efeitos benéficos demonstrados na literatura, o UST e outros tipos de eletroterapia ainda são técnicas subutilizadas em casos de reabilitação cirúrgica que poderiam melhorar de forma significativa a recuperação de 
feridas crônicas, com diminuição da necrose tecidual e da infecção local, e melhora da proliferação celular e da resposta imune (Rashidi et al., 2015; Alkahtani et al., 2017).

A maioria das informações disponíveis atualmente recomenda que a densidade de energia por sessão de tratamento esteja dentro da faixa de 0,1 a $12,0 \mathrm{~J} / \mathrm{cm}^{2}$ e que, em grandes condições de lesão tecidual, possa chegar até $30 \mathrm{~J} / \mathrm{cm}^{2}$, variando conforme o comprimento de onda e o diiodo utilizado (Viana et al., 2014; Rashidi et al., 2015; Diwan e Abid, 2016). No presente caso, aplicouse $20 \mathrm{~J} / \mathrm{cm}^{2}$ pontualmente, com comprimento de onda de $830 \mathrm{~nm}$, em uma ferida extensa e de curso crônico, o que permitiria, segundo a literatura, a utilização de dosagens acima de 12 e abaixo de $30 \mathrm{~J} / \mathrm{cm}^{2}$. Moraes et al. (2014) aplicaram o LBP também com comprimento de onda de $830 \mathrm{~nm}$ em varredura de 60 a $100 \mathrm{~J}$ total na ferida e $7 \mathrm{~J} / \mathrm{cm}^{2}$ nas bordas, e obtiveram resultados satisfatórios no processo cicatricial, reafirmando a possibilidade de utilização de dosagens superiores a $12,0 \mathrm{~J} / \mathrm{cm}^{2} \mathrm{em}$ grandes áreas de lesão tecidual.

De acordo com Alkahtani et al. (2017), o intervalo de 0,5 a $3 \mathrm{~W} / \mathrm{cm}^{2}$ do UST exerce resultados terapêuticos satisfatórios e efeitos adversos mínimos. As ondas promovidas pelo UST podem acelerar a velocidade de cicatrização de feridas abertas e crônicas, bem como lesões dos tecidos profundos. Efeitos semelhantes puderam ser constatados por Moraes et al. (2010, 2014a, b) e, ainda neste caso, com a utilização de baixas frequências de UST em intensidades variando de 0,5 a $1 \mathrm{~W} / \mathrm{cm}^{2}$ em feridas crônicas de equinos, obtendo-se excelentes resultados nos aspectos finais das feridas. Alkahtani et al. (2017) descrevem que para o desenvolvimento de um o protocolo clínico adequado, faz-se necessário maiores ensaios pré-clínicos e clínicos in vitro e in vivo para a doseresposta exata para cada tipo de ferida.

\section{Conclusão}

A combinação das duas técnicas fisioterápicas apresentou resultados satisfatórios, diminuindo consideravelmente o tempo de cicatrização, com interessante resultado estético final.

\section{Referências}

Alkahtani AS, Kunwar PS, Jalilifar M, Rashidi S, Yadollahpour A. Ultrasound-based Techniques as Alternative Treatments for Chronic Wounds: A Comprehensive Review of Clinical Applications. Cureus. 2017;9(12):e1952.

Araujo AL, Teixeira FA, Lacerda TF, Flecher MC, Souza VRC, Coelho CS. Effects of topical application of pure and ozonized andiroba oil on experimentally induced wounds in horses. Braz J Vet Res Anim Sci. 2017;54(1):66-74.

Celeste C. Selection of Suture Materials, Suture Patterns, and Drains for Wound Closure. In: Theoret C, Schumacher J (EE.). Equine wound management. 3 ed. Iowa: John Wiley \& Sons; 2017. p.173-99.

Chang YR, Perry J, Cross K. Low-frequency ultrasound debridement in chronic wound healing: a systematic review of current evidence. Plast Surg (Oaky). 2017;25(1): 21-6.

Diwan MF, Abid TA. The effect of different doses of 660 $\mathrm{nm}$ laser therapy on open skin wound healing in Wistar rats. Kufa J Vet Sci. 2016;7(2):130-7.

Moraes JM, Dumont CBS, Sales JVF, Bravo MO, Araújo PC, Pereira LC, et al. Ultrassom terapêutico como tratamento na cicatrização de feridas sépticas equinas. XI Conferência Anual da Abraveq; $12-13$ jun 2010; São Paulo, SP. Ribeirão Preto: Abraveq; 2010.

Moraes JM, Bravo MO, Mota ALAA, Dumont CBS, Pereira LC, Ximenes FHB, et al. Ultrassom terapêutico como tratamento na cicatrização de feridas em equinos. PUBVET. 2014a;8(16):Art.1760.

Moraes JM, Bravo MO, Huaixan LN, Villa Filho PC, Ximenes FHB, Teixeira Neto AR, et al. Ultrassom terapêutico e laser de baixa potência no tratamento de abscessos em equinos. PUBVET. 2014b;8(16):Art.1759.

Paganela JC, Ribas LM, Santos CA, Feijó LS, Nogueira CEW, Fernandes CG. Abordagem clínica de feridas cutâneas em equinos. Rev Port Cienc Vet. 2009;104(569-572): 13-8. 
Rashidi S, Yadollahpour A, Mirzaiyan M. Low level laser therapy for the treatment of chronic wound: clinical considerations. Biomed Pharmacol J. 2015;8(2):1121-7.

Viana LFS, Wenceslau AA, Costa SCL, Figueiredo MAF, Andrade FSSD, Ferreira ML. Tratamentos complementares para ferida com tecido de granulação exuberante em um equino - Relato de caso. Rev Bras Med Vet. 2014;36(4):417-20. 